\title{
CARCINOMA IN SITU Y EMBARAZO
}

\author{
Dr. Rubén Dario Guzmán Alandete* \\ TRABAJO PRESENTADO CON MOTIVO DE LAS BODAS DE PLATA DE LA \\ SOCIEDAD COLOMBIANA DE OBSTETRICIA Y GINECOLOGIA
}

Muchos son los conceptos contradictorios emitidos por los diversos autores en relación a los cambios histológicos y citológicos que se presentan en el cuello y en el frotis vaginal de la mujer gestante y aún, sobre la conducta que hay que seguir en estas pacientes una vez diagnosticado un ca in situ. Así vemos que mientras unos son partidarios de una conducta expectante, conservadora, otros son más radicales y preconizan un tratamiento quirúrgico en todos los casos.

Estos conceptos tan diametralmente opuestos estriban en que algunos autores creen que el embarazo es capaz de producir alteraciones cervicales y citológicas imposibles de diferenciar de un ca pre-invasivo, que según ellos pueden desaparecer al terminar el embarazo; en cambio otros consideran que éste no tiene el mágico poder de mimetizar el ca pre-invasivo y el diagnóstico de estas lesiones tienen valor durante el mismo.

Es bien sabido hoy en día, que durante el embarazo pueden presentarse una serie de modificaciones que son importantes de conocer adecuadamente para valorarlas y diferenciarlas de un proceso maligno incipiente. Nesbit del John's Hopkins
(1), describe una hiperactividad de la capa basal del epitelio del cuello uterino; una hiperplasia glandular traducida por un aumento e hipertrofia en el número y en el tamaño de las glándulas endocervicales y una epidermización del epitelio glandular - una metaplasia del epitelio columnar en epitelio escamoso estratificado. Otros como Nieburg y Clyman (2), conceptúan que los cambios epiteliales más frecuentes en el cuello de la mujer gestante se manifiestan por una hiperplasia y metaplasia; por lo tanto existe un aumento de la proliferación del epitelio escamoso estratificado y sobre todo una proliferación notable de las células basales, lo cual ocurre del $10 \%$ al $20 \%$ de las embarazadas; también existe edema; cierta displasia epitelial (grado intenso de atipia celular); pérdida incompleta de la polaridad. Según Frigerald y Hellman (3), en ocasiones el grado de hiperplasia de las células basales y el trastorno de la polaridad celular pueden tener tal intensidad que es un poco difícil diferenciarlo de un ca in situ. Insisten, que la pérdida completa de la estratificación, como la pérdida completa de la

\footnotetext{
* Profesor Asistente del Depto. de Obstetricia y Ginecología de la Universidad Nacional.
} 
CUADRO NN: 1

MODIFICACIONES DEL CUELLO UTERINO EN EL EMBARAZO

\begin{tabular}{|c|c|c|}
\hline Autor & Epitelio escamoso estratificado & Epitelio glandular \\
\hline NESBIT & Hiperactividad basal. & $\begin{array}{l}\text { Hiperplasia glan- } \\
\text { dular, epidermiza- } \\
\text { ción y metaplasia. }\end{array}$ \\
\hline NI9BURG y CLYMAN & $\begin{array}{l}\text { Hiperplasia, displasia y pérdida incompleta de la } \\
\text { poiaridad celular. Proliferación de las cólu!as ba- } \\
\text { sales } 110 \text { al } 29 \% \text { de las embarazadas). }\end{array}$ & Metaplasia. \\
\hline FRIGERALD y HELLMAN & $\begin{array}{l}\text { Hiperplasia basal y pérdida de la polaridad in- } \\
\text { tensa: puede semejar un ca in situ. Requisitos } \\
\text { indispensables: pérdida completa de la polaridad } \\
\text { y de la estratificación. }\end{array}$ & \\
\hline FLUHMANN & Proliferación marcada. & Secreción activa. \\
\hline BENSON & Hiperplasia e hipertrofia. & $\begin{array}{l}\text { Hiperplasia e } \\
\text { hipertrofia. }\end{array}$ \\
\hline ROSENTHAL & Proliferación basal. & \\
\hline
\end{tabular}

polaridad son requisitos previos e indispensables para hacer el diagnóstico de ca intraepitelial. Fluhmann (4), cree que durante el embarazo el cuello uterino experimenta una notable proliferación y secreción activa como consecuencia del intenso estímulo hormonal, pero pueden existir modificaciones propias de un ca preinvasivo. Benson (5), sostiene que en el cuello de la mujer embarazada pueden observarse cuadros de hipertrofia y de hiperplasia de elementos escamosos y glandulares, pero, sin embargo, en caso de existir un ca intraepitelial o invasivo no debe confundírselos con los cambios gestacionales naturales. Rosenthal y cols. (16), estudirano el cuello de la mujer gestante y concluyeron que existe un estímulo hormonal del epitelio, especialmente de la capa basal; que éste cambio no es exclusivo del embarazo, pues puede observarse cuando existe una estimulación estrogénica marcada, como en la recién nacida, en los casos de administración de estrógenos en la mujer post-meno- páusica, y en las mujeres con una hiperplasia quística del endometrio. Afirman que los cambios producidos no pueden diferenciarse del ca intraepitelial y que en algunos casos son reversibles.

Revisaremos los conceptos de los diferentes autores y por último presentaremos nuestra breve casuística:

Wespi y cols. (7), han descrito casos de regresión completa de cuadros de hiperactividad de las células basales e incluso de formaciones intraepiteliales semejantes al ca in situ, una vez finalizado el embarazo.

Epperson y cols. (8), Nesbit y cols. (9), han recopilado algunos casos de ca pre-invasivo con embarazo, que experimentaron regresión durante el puerperio. Además, estos últimos autores, están de acuerdo en conceptuar que el frotis vaginal tiende a presentar anormalidades que desaparecen al terminar la concepción. 
Novak (10), considera que aun cuando las displasias no son diferentes a las de las pacientes no embarazadas, pueden hacerse más atípicas durante el embarazo, pudiendo regresar en los meses del puerperio. Cree que esta regresión no suele ser completa y que con cada embarazo subsiguiente la displasia cervical puede comenzar en un nivel más atípico.

Danforth (11), conceptúa que las desviaciones apreciadas durante la gestación son de carácter hiperactivo y es partidario de una conducta conservadora frente a cualquier proceso canceroso equívoco o marginal del cuello uterino.

Campos y Soiheit (12), Hellman (13), Epperson y cols. (8), Nesbit y cols. (14), afirman repetidamente que el estímulo hormonal del embarazo puede conducir a un tipo de displasia y de carcinoma, que tiene una duración fugaz y que desaparece en el plazo de 6 meses después del parto. Aún más, los primeros autores consideran que es difícil o imposible diagnosticar con seguridad un carcinoma in situ durante la gestación. Fluhmann (4), no es partidario de estos conceptos y los considera peligrosos, pues podrían conducir a resultados desastrosos si tuviesen una amplia difusión.

Autores como Greene (15), Hamperl y Kaufmann (16), Marcus (17), Marsh y Fritzgerald (18), Moore y Gusberg (19), Peckham, Chung, Bayly y Benaron (20), no creen que las lesiones displásicas y los ca in situ que aparecen durante el embarazo regresen espontáneamente después del parto y han aportado pruebas evidentes de que la displasia y el ca preinvasor aparecidos durante la gestación se comportan exactamente, de la misma manera, que en la mujer no embarazada. Así, Peckham y cols.
(20) publicaron los resultados de la observación continua en 19 mujeres, en las cuales la biopsia del cuello uterino tomada en el ante-partum demostró ca in situ: en 16 de ellas $(84,2 \%)$ las lesiones persistieron en el puerperio. Concluyen que estas lesiones no pueden ser consideradas como cambios transitorios del embarazo. Greene y Peckham (15), Marsh y Fritzgerald (18), conceptúan que el $73 \%$ al $78 \%$ de los casos de ca in situ diagnosticados durante el embarazo son demostrados aun en el puerperio; afirman que la valoración del ca pre-invasor durante el embarazo presenta intrínsecamente las mismas dificultades diagnósticas que en la mujer no embarazada.

Richard ( 21 ), en un estudio de 75 casos de $\mathrm{Ca}$ intraepilelial y embarazo, de los cuales 66 fueron seguidos en su evolución, encontró que en el $78 \%$ persistió el ca in situ durante el puerperio y en 21 hubo regresión. Concluye que este carcinoma tiene el mismo significado en la mujer gestante como en la que no lo está.

Te-Linde (22) considera que en todas aquellas ocasiones en que Ilegamos a un diagnóstico certero de ca in situ en la mujer grávida, podemos confirmarlo después del parto.

Rad, Marczinke, Boyes y Fidler (23), presentan un estudio de 105 embarazadas con citología sospechosa - positiva, en donde 96 de estas pacientes tenían un carcinoma pre-clínico comprobado por biopsia de cuello. Afirman que la certeza de la citología vaginal durante el embarazo es más o menos la misma que en los casos de no embarazos, y que es de gran importancia descubrir y tratar rápidamente el ca intraepitelial sin postergarlo hasta el puerperio. Por otra parte Slate, Martin y Merrit (24), conceptúan que los frotis defi- 
nitivamente anormales durante el ante-partum, permanecen de esa manerá e indican la presencia de lesiones atípicas comparables, que varían entre una displasia y un ca pre-invasivo.

Ferguson y Cavanagh (25), sostienen que el embarazo no siempre es la causa de la positividad citológica; que existen pruebas muy dicientes de que el ca in situ en la mujer embarazada no difiere del de la mujer no grávida. Igual opinión comparte Rad y cols. (23).

Ayre (26), Fluhmann (4), Spujt y cols. (27), afirman que la regresión de las lesiones típicas del $\mathrm{Ca}$ in situ durante o después del embarazo es rara y solamente la han visto cuan- do el cuello ha sido sometido previamente a una biopsia, tomada durante las investigaciones en el antepartum $\circ$ a cauterizaciones.

Nieburg y Clyman (20), conceptúan que muchas lesiones que desaparecen después del parto se debe a que en ellas faltó el criterio necesario para hacer el diagnóstico de $\mathrm{Ca}$ in situ; afirman que los cambios gravídicos semejantes a la hiperplasia de las células basales o a la displasia suelen sufrir regresión en el puerperio en el $95 \%$, mientras que la mayor parte del $\mathrm{Ca}$ intra-epitelial persistirán en el $80 \%$; por otra parte sostienen que es obligatorio descartar un ca invasivo del cuello uterino.

\section{CUADRO № 2}

FRECUENCIA

\begin{tabular}{lccc}
\hline \multicolumn{1}{c}{ Autor } & No de casos & No de embarazos & Porcentaje \\
\hline SPUJT y cols. (27) & 7 & 3.000 & $2,3 \%$ \\
HIRST (28) & 1 & 3.800 & $2,4 \%$ \\
SCHMITZ, ISAACS, FETHERSTON (29) & 13 & 10.369 & $1,2 \%$ \\
CUYLEN y cols. (30) & 17 & 1.451 & $0,00091 \%$ \\
NIEBURG y CLYMAN (2) & 6 & 65.148 & \\
IMI (Bogotá) & & & \\
En general la frecuencia oscila del $2,3 \%$ al $2,4 \%$. &
\end{tabular}

Entre nosotros Arévalo y Lozano en 1965 (31), en un estudio sobre Malignidad y embarazo presentan una incidencia de $0,031 \%$ de ca de cérvix invasivo y ningún caso de ca in situ en 135.479 mujeres embarazadas atendidas en 7 años en el Instituto Materno Infantil "Concepción Villaveces de Acosta" de Bogotá, pero durante ese lapso la citología vaginal en el pre y post-natal era de poca ocurrencia. Creemos que con la generalización de los estudios citológicos en la mujer gestante éstas consultas, aumentará la incidencia del hallazgo de esta afección.

\section{CUADRO № 3}

EDAD

\begin{tabular}{llrl}
\hline 3ENSON ( 5) & $30-40$ & años \\
MUC DUFF y cols. (30) & 32 & años \\
NILLIAMS (32) & $30-40$ & años \\
MI (Bogotá) & $26-42$ & años \\
\hline
\end{tabular}

Parece confirmado que el ca in situ del cuello uterino aparece en mujeres cuya edad es de 9 a 10 años menor de aquellas en que suele aparecer el ca invasivo, lapso que permite hacer un diagnóstico cuidadoso y una terapéutica definitiva. 


\section{Pesquisa del Carcinoma in situ de la embarazada}

Es demasiado difícil hacer el diagnóstico clínico del ca intra-epitelial en la mujer embarazada. Los antecedentes tienen poco valor; a la inspección del cuello uterino puede no apreciarse lesión o quizá una pequeña erosión aparentemente benigna; es más, existen casos de cervicitis crónicas que tienen una apariencia macroscópica más patológica que la de un ca intra-epitelial, de ahí la importancia que tienen los estudios citológicos vaginales. Por medio de este procedimiento en las consultas de Pre-natal y Post-natal, se pueden detectar un gran número de ca pre-clínicos que podrían pasar inadvertidos debido al buen aspecto del cuello uterino; de ahí que estamos convencidos, al igual que otros autores, que este procedimiento debe imponerse con carácter obligatorio y rutinario en estas consultas, pues además de proporcionarnos un reconocimiento de la lesión maligna, procura una excelente oportunidad para iniciar a nuestras mujeres en un hábito que durante toda la vida las conduzca a someterse a repetidos exámenes citológicos. Novak (10), considera que la primera visita es el mejor momento para obtener una muestra citológica, pues las infecciones cervicales aumentan a medida que avanza el embarazo con el consiguiente oscurecimiento y degeneración de las muestras celulares; también dá más oportunidad para que desaparezca la inflamación, a parte de poderse tomar cualquier medida diagnóstica 0 terapéutica. El ca intra-epitelial básicamente es asintomático; en algunas ocasiones las pacientes consultan por discreta hemorragia - vaginal o por flujo muy escaso. Montgomery T. L. (33), opina que la demora diagnóstica del ca del cuello uterino es casi dos veces más tardío en las grávidas que en las no grávidas. Según Juan León (30), el carcinoma de -cuello puede pasar inadvertido por varias razones: la primera, es que sus manifestaciones son interpretadas erróneamente y así las hemorragias que se observan, en el $73 \%$ de los casos son atribuidas en el primer trimestre a una amenaza de aborto o a un embarazo ectópico y en el último trimestre a la placenta previa o al desprendimiento prematuro de la placenta. Lo mismo ocurre con las secreciones vaginales sanguinolentas acuosas o purulentas que se registran en el $19 \%$ de los casos, las cuales suelen atribuirse a una tricomoniasis vaginal, moniliasis, etc. La segunda razón, es que se elude en ellas el tacto vaginal y por supuesto, la inspección del cuello uterino con espéculo.

En el post-natal es obligatorio antes de proceder a cauterizar una cervicitis crónica o de reparar un desgarro cervical producidos por el parto $\circ$ por instrumentaciones, tomar una citología vaginal.

\section{Conducta a seguir en una paciente embarazada de acuerlo al examen clínico y los hallazgos citol gicos}

\section{(Ver Cuadro No 5).}

Toda pcaiente que consulte a Prenatal, aparte de un buen examen ginecológico con especuloscopia, se le debe practicar una toma de citología vaginal. Si la citología es sospechosa debe investigarse si existe leucorrea o una vagino-cervicitis; en caso afirmativo se debe tratar tales afecciones y tomar nueva citología vaginal aproximadamente al mes. En caso de lesión cervical aparente y citología positiva debe tomarse una biopsia cervical; si la biopsia es positiva para ca in situ se impone la conización; si la biopsia es negativa se so- 


\section{CUADRO NN 4}

\section{CA. PREINVASIVO Y EMBARAZO}

WESPI y col. EPPERSON y col. NESBIT y col. CAMPOS, SCIHEIT, HELLMAN

\section{ROSENTHAL}

AYRE, FLUHMANN, SPUJT y col.

NIEBURG Y CLYMAN

SLATE, MARTIN y MERRIT

FERGUSSON y CAVANAGH, RAD y col.

GREENE, HAMPERL Y KAUFMANN, RAD, MARCUS, MARSH Y FITZGERALD, MOORE, GUSBERG, CHUNG, PECKHAM, BAYLY, BENARON, RICHARD.

TE-LINDE.

MARCZINKE, BOYES, FIDLER.
Algunos casos; igualmente de frotis vaginal anormal.

Displasia y Ca. fugaz que desaparecen en 6 meses. Difícil o imposible diagnosticar con certeza un $\mathrm{Ca}$. in situ en la mujer gestante.

Algunos casos.

En pocos casos, por biopsia o cauterio.

Diagnóstico insuficiente. La hiperplasia de la capa basal, y la displasia regresan en un $95 \%$.

No; igualmente los frotis vaginales definitivamente anormales durante el anteparto indicarán una displasia o un Ca. preinvasivo.

Muy raro que regresen

Muy raro.
Cuando menos en un $80 \%$.

El embarazo no siempre es la causa de la po sitividad citológica. El Ca. in situ en la mujer embarazada no difiere del de la mujer no grávida.

Del $73 \%$ al $84,2 \%$ de los Ca. in situ son de mostrables en el puerperio. El Ca. y las displasias se comportan como en la no embarazada.

La certeza de la citología diagnóstica ciurante el embarazo es más o menos la misma que en la mujer no embarazada. 
CUADRO N: 5

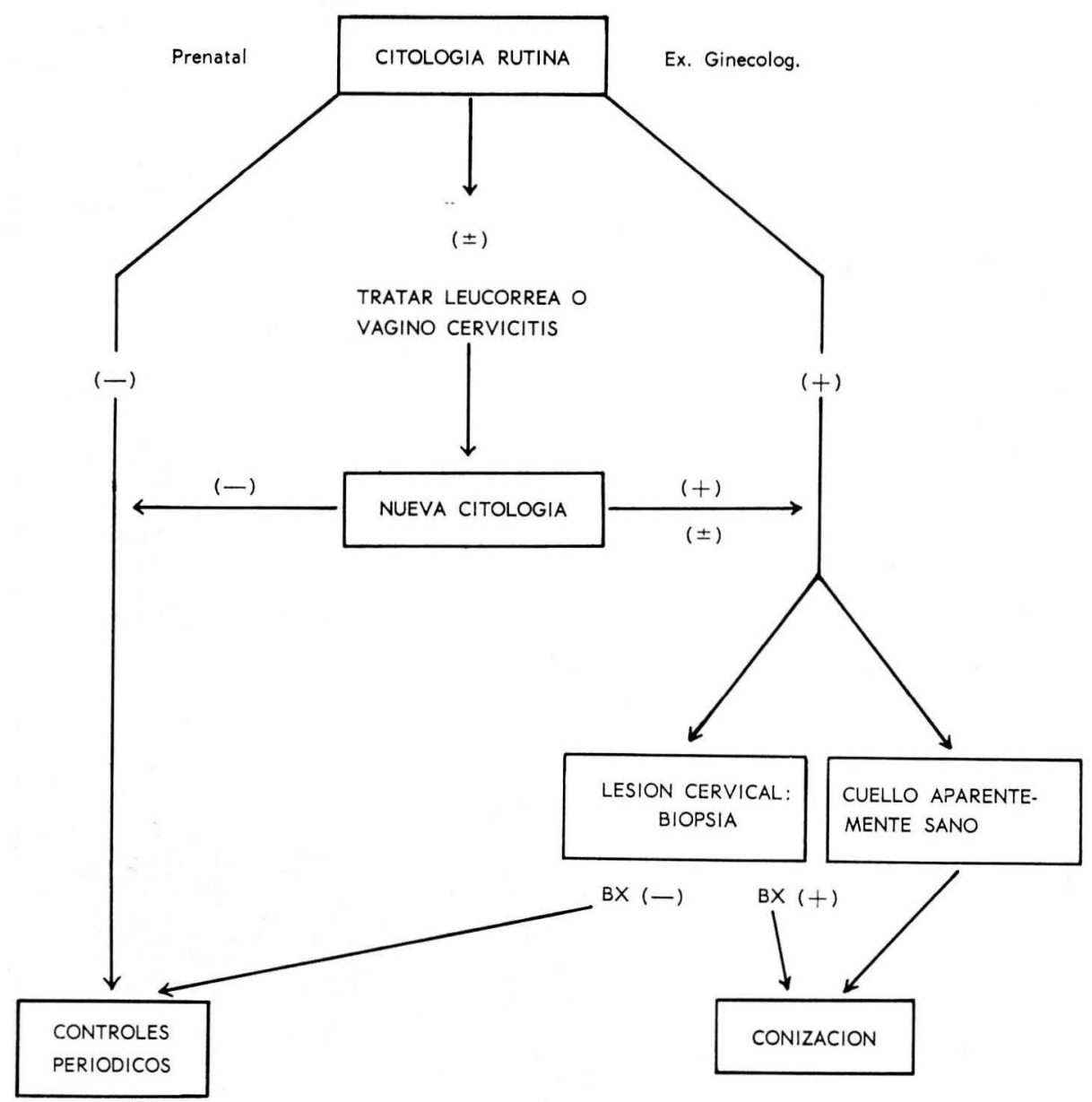

meterá la paciente a controles periódicos. Si la citología es positiva y el cuello es aparentemente sano también se impondrá la conización.

\section{Tratamiento}

Williams (32) conceptúa que cuando se hace el diagnóstico de ca in situ durante el embarazo y se tiene la seguridad de que la lesión es verdaderamente un ca intra-epitelial, con- viene dejar que el embarazo prosiga; que el parto se efectúe normalmente; la vigilancia ulterior debe ser rigurosa.

Novak y Galvin en 1951 y Jones en 1952, citados por Juan León (30), aconsejan no recurrir a medidas terapéuticas radicales, en especial a operaciones, hasta tanto se confirme que se está realmente en presencia de un ca pre-invasor. Por lo tanto, 
CUADRO N 6

tRATAMIENTO DEL CA. PREINVASIVO EN EL EMBARAZO

\begin{tabular}{|c|c|c|c|}
\hline Autor & Conización & Parto & Puerperio \\
\hline $\begin{array}{l}\text { WILLIAMS, NOVAK y GALVIN, JO- } \\
\text { NES, DEAN, ISBELL, WOODARD, } \\
\text { MCKELWAY, JORDAN y DARNALT } \\
\text { (Colombia). }\end{array}$ & No conización. & Parto normal. & Investigar. \\
\hline $\begin{array}{l}\text { FLUHMANN, OSBAND, RAD, NI- } \\
\text { CHOLSON, PARKER y col. MARC- } \\
\text { ZINKE, BOYES, FIDLER. }\end{array}$ & $\begin{array}{l}\text { Si practican biopsias y conizacio- } \\
\text { nes como en la no gestante. }\end{array}$ & & \\
\hline FESTE， TOPEK， SKOGLAND. & Si, hasta la $35 a$. semana. & & \\
\hline $\begin{array}{l}\text { OSBAND, JONES, SCHMITZ, } \\
\text { ISAACS, FETHERSON. }\end{array}$ & $\begin{array}{l}\text { Sí, hasta la } 34 a \text {. o la } 35 a \text {. se- } \\
\text { mana. }\end{array}$ & & \\
\hline GALLEGOS (Venezuela). & Si, hasta la $34 a$. semana. & $\begin{array}{l}\text { Parto normal si la citología es } \\
\text { negativa. }\end{array}$ & $\begin{array}{l}\text { Investigar, si no se ha practica- } \\
\text { do conización en el anteparto. }\end{array}$ \\
\hline GONZALEZ LAYA y col. (México). & Sí, hasta la 36a. semana. & Parto normal post-conización. & $\begin{array}{l}\text { Investigar, si no se ha practicado } \\
\text { conización en el anteparto. }\end{array}$ \\
\hline $\begin{array}{l}\text { FERGUSSON, MARCUS, MOORE, } \\
\text { WELLS, MUSSEY, DECKER Y TAY- } \\
\text { LOR. }\end{array}$ & Si, hasta la $36 a$. semana. & & \\
\hline CAVANAGH y OFFEN. & $\begin{array}{l}\text { Sí, desde la } 8 a . \text { hasta la } 38 a . \\
\text { semana. }\end{array}$ & & \\
\hline LAPID， DAVIDS， GUSBERG. & $\begin{array}{l}\text { Sí, desde la } 2 \frac{1}{2} \text { hasta la } 36 a \text {. } \\
\text { semana. }\end{array}$ & Parto normal post-conización. & $\begin{array}{l}\text { Investigar, si no se ha hecho co- } \\
\text { nización en el anteparto. }\end{array}$ \\
\hline KAPLAN, KAUFMAN. & Sí, $2^{o}$ y $3 e r$. trimestre. & Parto normal post-conización. & $\begin{array}{l}\text { Investigar, si no se ha practicado } \\
\text { conización en el anteparto. }\end{array}$ \\
\hline IMI (Bogotá). & $\begin{array}{l}\text { Si, hasta la } 36 a \text {. semana. Des- } \\
\text { pués de la } 36 a \text {. semana: opcio- } \\
\text { nal. }\end{array}$ & $\begin{array}{l}\text { Parto normal si la citología es } \\
\text { negativa. } \\
\text { Cesárea segmentaria con citología } \\
\text { sospechosa o positiva si no se } \\
\text { conizó o cuando el embarazo es- } \\
\text { tá avanzado y sobreviene el tra- } \\
\text { bajo de parto antes de } 10 \text { días } \\
\text { post-conización. }\end{array}$ & $\begin{array}{l}\text { Investigar, si no se ha practicado } \\
\text { conización en el anteparto. }\end{array}$ \\
\hline
\end{tabular}


dejan evolucionar el embarazo hasta su final, vigilando cuidadosamente la situación para estimarla finalmente durante el puerperio.

Mekelway, citado por Arévalo y Lozano (31), no es partidario de la conización en la mujer gestante por la hemorragia que pueda presentarse.

Dean, Isbell, y Woodard (34), en caso de citologías persistentes, practican biopsia y si llegasen a comprobar el carcinoma in situ, tratan estas pacientes de una manera conservadora permitiendo el parto por vía vaginal a menos que existiese una indicación obstétrica. A los 4 meses siguientes toma nuevas citologías y practican histerectomía en caso de persistencia de la afección.

En nuestro medio Jordan y Darnalt en 1955 (35), aconsejan esperar la evolución del embarazo a su término, vigilando estrechamente a la paciente, repitiendo citologías y biopsias en caso de ser necesarias; posteriormente en el puerperio, si persiste el cuadro histológico, recomiendan un tratamiento final, según la edad de la enferma.

Darnalt en 1966 (36), es partidario de tomar citologías periódicas, biopsias múltiples durante el embarazo con el fin de aclarar un diagnóstico; no e spartidario de la conización por el riesgo de hemorragia que podría presentarse.

Por otra parte, estudios como los de Fluhmann (4-, demuestran que para el tratamiento del ca in situ y de la displasia cervical, rigen los mismos principios tanto para la mujer embarazada como para la que no lo está. Sostiene que durante el embarazo pueden efectuarse biopsia múltiple, conizaciones, sin poner en peligro el embarazo si se toman las debidas precauciones.
Osband y Nicholson (37), practican conización con el fin de confirmar el diagnóstico de ca pre-invasivo, permitiendo el parto por vía vaginal; en el post-partum aconsejan la histerectomía si el caso lo requiere.

Parker y cols. (38) toman biopsia, practican conización en cualquier mujer embarazada que tenga citología atípicas y una lesión del cuello uterino clínicamente sospechosa de malignidad.

Pionero de estos conceptos es Fluhmann (4), quien afirma que el hallazgo de dos o más frotis vaginales positivos o de una biopsia positiva para ca in situ, exige una conización con el fin de eliminar la presencia de una lesión invasora. Si la conización revela una lesión de esta índole, permite que continúe el embarazo hasta el término y en fecha posterior toma la decisión final sobre la conveniencia de una histerectomía.

Rad y cols. (23), aconsejan la conización en el segundo y tercer trimestre del embarazo. Además, dá las siguientes recomendaciones para su tratamiento: si en la conización se encuentra una displasia cervical, no se requiere nada más; en caso de encontrarse un ca in situ que es completamente removido por la conización, tampoco se hace más, excepto un control estricto en el futuro. Si la lesión es in situ pero se considera que estuvo incompletamente removida por la conización, las investigaciones son postergadas hasta que termina el embarazo; si en estas investigaciones ulteriores, la citología e saún sospechosa o positiva, el cuello podrá ser reconizado si la mujer desea tener más hijos o podrá ser histerectomizada en caso contrario. Si en la conización se encuentran focos microscópicos de invasión, 
el tratamiento depende del tamaño del cono y de la extensión de la lesión. Aconseja, para estos casos, resolverlos individualmente, pudiendo intentarse la reconización con el fin de extirpar completamente la lesión si se cree que estuvo incompletamente extirpada por la conización inicial; en otros casos puede estar indicada la terminación del embarazo por medio de una histerectomía y por último puede llevarse el embarazo hasta su término y manejarse como un ca in situ. Cuando se encuentra una invasión oculta en la conización, se terminará el embarazo y se trata la paciente como un estado 1 de carcinoma invasivo del cuello uterino. En su trabajo presentan 38 casos de conización con embarazo, 4 de los cuales terminaron en abortos y 1 presentó una hemorragia tardía diez días después de la conización; consideran que el riesgo de complicaciones es bajo y vale la pena correrlos.

Feste, Kaufman, Skogland, Topek (39), proponen conización antes de las 35 semanas de embarazo. Gallegos en Venezuela (40), es partidario de la conización antes de la 34a semana del embarazo. Presenta 12 casos de conizaciones (seis en el primer trimestre y seis en el segundo trimestre), dos de los cuales presentaron hemorragia abundante; en ningún caso hubo interrupción del embarazo, ni complicaciones en el parto.

González Loya y cols. (41) en México, practican conización hasta la 36. semana en la mujer embarazada, teniendo todos los cuidados pre, trans y postoperatorios habituales en cirugía y embarazo; igual opinión comparte Ferguson (42), Marcus (17), Moore y Wells (43), Mussey y Decker (44), y Taylor (45). Estos últimos autores citados, son partidarios de dejar evolucionar el parto por vías naturales, después de la conización, efectuando la cesárea solo por alguna otra indicación obstétrica.

Ferguson y Cavanagh (25), y Offen (46), practican conización en la mujer embarazada desde la 8a hasta la 38. semana de embarazo. Lapid, Davids y Gusberg (47), afirman que conviene hacer biopsia y conización, si están indicados. Han efectuado esta última intervención incluso si el embarazo tiene dos y media semanas de duración o treinta y seis. Sostienen que es mejor hacer diagnóstico preciso lo más temprano posible en el embarazo, pues habrá que hacer terapéutica radical si se descubre cáncer cervical.

Osband R., Jones W. (37); Schmitz H. E., Isaacs y J. H. y Fetherston W. (29) proponen biopsia, conización o ambos métodos hasta la $34^{\text {a }}$ o 35 a semana de embarazo. Conceptúan además, que este último método no es difícil ni altera la evolución del embarazo.

En el Instituto Materno Infantil "Concepción Villaveces de Acosta" de Bogotá, somos partidarios de efectuar conización del cuello uterino en la mujer gestante hasta la 36a semana de gestación debido a las modificaciones cervicales presentes a esa edad del embarazo, sin embargo habrá casos en los cuales sí se pueda practicar en edad más avanzada si las modificaciones del cuello no son muy manifiestas. En caso de no haberse efectuado la conización después de la 36: semana, bien sea por falta ed control adecuado de la paciente, o bien sea por la edad en que evoluciona el embarazo, aconsejamos practicar la cesárea segmentaria, ya que es imposible de tener el 100\% de seguridad en el diagnóstico a base de estudios citológicos vaginales de descartar de una manera conclu- 
CUADRO N: 7

CASUISTICA

\begin{tabular}{|c|c|c|c|c|c|c|c|c|c|c|c|c|c|}
\hline Caso & H. Clíniea & Edad & Gravidez & Semanas & $\begin{array}{l}\text { Citología } \\
\text { Prevía }\end{array}$ & Estado del Cuello & Conización Anteparto & $\begin{array}{l}\text { Citología } \\
\text { Anteparto }\end{array}$ & Conducta Obstétrica & Niño & Puerperio & Anat. Pat. Final & Contr. \\
\hline 1 & 117135 & 28 & $G: 5 P: 4$ & 39 & $\begin{array}{c}\text { IV V } \\
\text { (Prenatal) }\end{array}$ & $\begin{array}{l}\text { Erosión. } \\
\text { Bx: Ca in Situ. }\end{array}$ & & & $\begin{array}{l}\text { Ingresa en trabajo } \\
\text { de parto: Cesárea } \\
\text { por Sit. transversa. }\end{array}$ & $\begin{array}{l}\text { Fem. } 4 / 10 \\
3.030 \mathrm{grs}\end{array}$ & Conización. & $\begin{array}{l}\text { Cervicitis Cron. } \\
\text { (Conizac.) }\end{array}$ & Bien \\
\hline 2 & 84777 & 42 & $G: 9 P: 9$ & 8 & $\checkmark$ & $\begin{array}{l}\text { Erosión. } \\
\mathrm{Bx}: \mathrm{Ca} \text { in Situ. }\end{array}$ & $\mathrm{Ca}$ in situ & & $\begin{array}{l}\text { Raspado Uterino } \\
\text { por aborto. }\end{array}$ & & & $\begin{array}{l}\text { Ca in situ. } \\
\text { (Conizac.) }\end{array}$ & Bien \\
\hline 3 & 224057 & 37 & $\begin{array}{c}G: 9 P: 7 \\
A: 1\end{array}$ & 43 & IV & $\begin{array}{l}\text { Aparentemente } \\
\text { sano. }\end{array}$ & $\begin{array}{l}\text { A las } 8 \text { semanas: } \\
\mathrm{Ca} \text { in situ }\end{array}$ & 111 & $\begin{array}{l}\text { Cesárea e Hist. } \\
\text { Abd. ampliada. }\end{array}$ & $\begin{array}{l}\text { Fem. } 4 / 10 \\
3.200 \text { grs. }\end{array}$ & & $\begin{array}{l}\text { Ca in situ Resi- } \\
\text { dual (Hist.) }\end{array}$ & Bien \\
\hline 4 & 66828 & 26 & $G: 5 P: 4$ & 20 & $\begin{array}{c}V \text { IV } \\
\text { (Prenatal) }\end{array}$ & $\begin{array}{l}\text { Aparentemente } \\
\text { sano. }\end{array}$ & $\begin{array}{l}\text { A las } 20 \text { semanas: } \\
\mathrm{Ca} \text { in situ }\end{array}$ & 1 & & & $\begin{array}{l}\text { Muerte in Utero } \\
2 \text { meses más } \\
\text { tarde. Inducción. }\end{array}$ & $\begin{array}{l}\text { Ca in situ } \\
\text { (Conizac.) }\end{array}$ & Bien \\
\hline 5 & 168178 & 36 & $G: 6 P: 5$ & 38 & $\begin{array}{c}\text { V } \\
(\text { H.S. de D. })\end{array}$ & $\begin{array}{l}\text { Ectropión } \\
\text { labio posterior. }\end{array}$ & $\begin{array}{l}\text { A las } 14 \text { semanas: } \\
\mathrm{Ca} \text { in situ residual. }\end{array}$ & $\| \mathrm{IV}$ & $\begin{array}{l}\text { Cesárea e Hist. } \\
\text { Abd. ampliada. }\end{array}$ & $\begin{array}{l}\text { Fem. } 6 / 10 \\
2.660 \text { grs. }\end{array}$ & & $\begin{array}{l}\text { Ca in situ Resi- } \\
\text { dual (Hist.) }\end{array}$ & Bien \\
\hline 6 & 81821 & 39 & $G: 7 P: 6$ & 36 & $\begin{array}{c}\text { V IV } \\
\text { (Prenatal) }\end{array}$ & $\begin{array}{l}\text { Aparentemente } \\
\text { sano. }\end{array}$ & $\begin{array}{l}\text { A las } 36 \text { semanas: } \\
C a \text { in situ. }\end{array}$ & 11 & $\begin{array}{l}\text { Cesárea e Hist. } \\
\text { Abd. ampliada. }\end{array}$ & $\begin{array}{l}\text { Fem. } 10 / 10 \\
2.400 \mathrm{grs}\end{array}$ & & $\begin{array}{l}\text { No tumor resi- } \\
\text { dual (Hist.) }\end{array}$ & Bien \\
\hline
\end{tabular}


yente la existencia de un ca invasor, en el cual el traumatismo del parto y el puerperio serían de consecuencias funestas. En tales situaciones, de acuerdo con la mayoría de los autores, aconsejamos reanudar el estudio a partir de la 4a o 6a semana del puerperio, a base de citologías, biopsia, conización, lo cual nos descartará o afirmará el ca invasor; o una regresión de una hiperplasia de células basales o un carcinoma in situ que es poco probable que regrese al estado normal del epitelio.

Somos partidarios también, de una citología antes del parto; si es negativa puede permitirse el parto por las vías naturales. Se practicará cesárea cuando la citología postconización resultare positiva o sospechosa; también cuando la conización es realizada inmediatamente antes del parto y la citología es sospechosa. El criterio se basa para ello por haber encontrado un $3 \%$ de ca invasivo cerca del orificio interno y el peligro de diseminación que ocasionaría dejar evolucionar el parto por las vías naturales.

Cuando se trata de mujeres cerca de la menopausia o en las grandes multíparas, aconsejamos practicar como tratamiento final cesárea e histerectomía abdominal ampliada en el mismo acto operatorio o esta última intervención unos días más tarde. Al hacer la histerectomía total abdominal debe extirparse una buena porción de vagina; la extirpación o conservación de los anexos se decidirá según la edad de la paciente y la observación o falta de enfermedad pélvica asociada. No consideramos necesario practicar la histerectomía abdominal con linfadenectomía, ya que es un procedimiento innecesario $y$ agresivo para esta clase de carcinoma.
A aquellas pacientes a quienes como tratamiento final se le ha practicado conización serán motivo de controles periódicos ginecológicos y citológicos en el puerperio y después del mismo.

\section{Casuística (Ver Cusiro 7)}

Como puede apr se existen conductas erradas debido a que aún no teníamos un criterio definido sobre este problema.

1er. CASO: B.M. Hx. 117135. Edad: 28 años. $G: 5$ : 4. Actualmente cursa un embarazo de más o menos 39 semanas. Citologías vaginales tomadas en la consulta Prenatal: A.4 y A.5. Estado del Cuello: erosionado. Biopsia del cuello: $\mathrm{ca}$ in situ. Ingresa al 1 . M.I. en trabajo de parto, con un feto vivo en situación transversa. Se le practica cesárea por situación transversa, obteniéndose un feto vivo, femenino, con un Apgar de 7/10. de 3.030 grs. En el puerperio se le practica conización, cuyo resultado fue de cervicitis crónica.

Comentario: No se conizó antes del parto por haber ingresado al hospital en trabajo de parto. Se optó por la conducta de cesárea por tratarse de un feto en una situación distócica. La conización en el puerperio dio una cervicitis crónica, lo cual nos hace pensar que el carcinoma in situ fue extirpado en la biopsia anterior. Controles citológicos y clínicos: normales.

29 CASO : O.V. Hx: 84777. Edad: 42 años. G: 9. P: 8a: 1. Presentaba un embarazo de más o menos 8 semanas. Citología vaginal: A5. Estado del cuello: erosionado. Ingresa al I.M.I. por presentar un aborto incompleto. Se le practica raspado uterino y biopsia de cuello, cuyo resultado anatomopatológico mostró un ca in situ. Se practica conización, la cual es positiva para ca in situ.

En el puerperio presentó un accidente cerebro-vascular, motivo por el que no se histerectomizó.

Comentario: Paciente a quien se le descubrió un ca in situ en el momento del raspado uterino por aborto incompleto; se ratificó el diagnóstico por medio de la conización. No se le practicó histerectomía abdominal ampliada, conducta que era la más indicada por tratarse de una paciente de más de 40 años y gran multípara, debido al accidente cerebrovascular presentado en el puerperio, del cual 
se recuperó paulatinamente. Controles citológicos y clínicos: normales.

3er. CASO: A.G. de M. Hx: 224057. Edad: 37 años. G: 9. P: 7. A: 1. Actualmente cursa un embarazo de más o menos 40 semanas. Antecedente de habérsele practicado una conización cuando tenía 8 semanas de embarazo por presentar varias citologías A.5 y una biopsia de cuello positiva para ca in situ. El resultado de la conización fue concluyente para ca pre-invasivo.

Ingresa al I.M.I. con un feto vivo, en situación longitudinal, presentación de vértice. Citología ante-partum: A3. A la especuloscopia el cuello se encuentra aparentemente sano. Se le practica cesárea e histerectomía abdominal ampliada, obteniéndose un feto vivo, femenino, de 3.200 grs. con un Apgar de 4/10.

Anatomía patológica del útero: $\mathrm{Ca}$ in situ residual.

Comentario: Consideramos que este caso no fue bien manejado pues la citología ante-partum fue sospechosa, lo cual habría indicado practicar una cesárea y posteriormente en el puerperio reanudar el estudio del cuello; ha podido tratarse de un ca invasivo. El examen anatomopatológico del útero mostró ca in situ residual, lo cual sugiere que la conización no fue adecuadamente amplia.

Controles citológicos y clínicos: normales.

49 CASO: F.G. de G. Hx: 86828. Edad: 26 años. G: 5. P: 4. Embarazo actual de más 0 menos 20 semanas. Dos citologías practicadas en la consulta de Prenatal: A.5 y A.4. A la especuloscopia el cuello se encuentra aparentemente sano. Se le practica conización sin ninguna complicación, cuyo estudio fue positivo para ca in situ. Vuelve a control dos meses más tarde con un feto muerto y retenido; la citología de control: A.1. A la inspección el cuello continúa aparentemente sano. Se le induce en tres oportunidades obteniéndose un feto muerto y macerado.

Comentario: Vemos en este caso, la importancia de la citología vaginal rutinaria, en donde se pudo detectar un carcinoma en su comienzo, diagnóstico que fue corroborado por la conización. Desgraciadamente dos meses más tarde el feto murió in útero.

Controles clínicos y citológicos: normales.

59 CASO: B.O. $\mathrm{Hx}:$ 168178. Edad: 36 años. G: 6. P: 5. Citología vaginal rutinaria: A.5. Estado del cuello: discreto ectropión del labio posterior. Biopsia del cuello: ca in situ con severo compromiso de las glándulas endocervicales. Se le coniza a las 14 semanas de gestación. Resultado del cono: ca in situ; se ve tumor residual en la línea de resección.
Dos controles citológicos post-conización fueron A.2. A las 38 semanas de embarazo se le toma una nueva citología vaginal cuyo resultado fue A.5. Se le practica cesárea e histerectomía abdominal ampliada, obteniéndose un feto vivo, femenino, de 2.660 grs. con un Apgar de 6/10. Anatomía patológica del útero: $c a$ in situ residual.

Comentario: Paciente a quien se le descubrió el ca in situ por la citología vaginal rutinaria. La biopsia y la conización fueron positivas para ca in situ; desgraciadamente quedó tumor residual en el cono. Una citología a las 38 semanas mostró A.5. Se le practicó cesárea e histerectomía abdominal ampliada, conducta errada, pues se le ha debido practicar solamente cesárea y posteriormente estudiar el cuello en el puerperio, ya que si hubiera sido un ca invasor el tratamiento sería in. suficiente.

69 CASO: H. de G. Hx: 81821. Edad: 39 años. G: 7. P: 6. Paciente a quien se le practica en la consulta de Prenatal dos citologías vaginales rutinarias, cuyos resultados fueron A.5. y A.4. sucesivamente. En ese entonces presentaba un embarazo de aproximadamente 35 semanas. Estado del cuello: sano. Se le practica conización a las 36 semanas, sin complicaciones; el examen del cono fue positivo para ca in situ. Citología post-conización: A.2. A las 38 semanas se le practica cesárea e histerectomía abdominal ampliada, obteniéndose un feto vivo, femenino, de 2.400 grs. con Apgar de $10 / 10$.

Resultado anatomopatológico del útero: No hay tumor residual.

Comentario: Paciente a quien se le descubrió un ca in situ por la citología vaginal rutinaria, a pesar de presentar un cuello aparentemente sano. Se le sometió primero a conización a las 36 semanas de embarazo, cuyo resultado del cono fué positivo para ca in situ y posteriormente se le practicó cesárea e histerectomía abdominal ampliada por tratarse de una gran multípara con 39 años de edad, conducta que compartimos por ser la más lógica y adecuada al caso, aunque se le ha podido permitir el parto por las vías naturales.

Controles citológicos y clínicos: normales.

\section{Resumen y Comentarios}

1. Se hace una revisión de los conceptos emitidos por los diferentes autores, en relación con los cambios histológicos y citológicos que suelen presentarse en el cuello y en el fro- 
tis de la mujer embarazada. Somos partidarios de los conceptos de aquellos autores, quienes tratan de demostrar que el embarazo no tiene el mágico poder de mimetizar el carcinoma preinvasivo, y que los casos de carcinoma intraepitelial diagnosticados durante la gestación tienen el mismo valor que en la no embarazada. También estamos de acuerdo en que los frotis vaginales definitivamente anormales durante el embarazo permanecerán de esa manera en el post-partum. Creemos que el embarazo no siempre es la causa de la positividad citológica, y estamos plenamente identificados con aquellos autores quienes afirman que la regresión de las lesiones típicas del ca in situ es rara.

2. Su frecuencia es muy variada; en general es del $2,3 \%$ al $2,4 \%$. En el I.M.I. es aproximadamente de 1 caso de ca in situ por cada 10.000 embarazos.

3. Hacemos hincapié en la importancia del examen clínico y citológi$c o$ en las consultas de Prenatal $y$ Post-natal, en donde se puede descubrir un buen número de carcinomas in situ del cuello uterino que hubieran podido pasar inadvertidos por encontrarse un cuello sano o aparentemente sano. Creemos que la citología vaginal rutinaria es un procedimiento que debe imponerse con carácter obligatorio en todos los centros de maternidad.

4. La edad en que se presenta esta afección es muy variada. Sin embargo, se admite que suele aparecer 9 a 10 años antes de la edad promedio del ca invasor.

5. Hacemos una revisión de los conceptos sobre tratamiento de estas enfermas. Estamos de acuerdo con quienes toman biopsias, practican conización, en cualquier mujer emba- razada que tenga citologías atípicas y un cuello aparentemente sano, o con una lesión clínicamente sospechosa de malignidad.

6. Somos partidarios de practicar conización hasta las 36 semanas de gestación, salvo aquellos casos en los cuales las modificaciones cervicales del embarazo no son tan marcadas, pudiendo efectuar un poco más tarde. Dejemos evolucionar el parto por las vías naturales después de la conización, siempre y cuando la citología vaginal ante-partum sea negativa, efectuando la cesárea solo por alguna indicación obstétrica. En caso de no haberse efectuado la conización antes de la semana 36, aconsejamos practicar cesárea segmentaria, ya que es imposible de tener el 100\% de seguridad en el diagnóstico a base de estudios citológicos vaginales de descartar un carcinoma invasor, el cual el traumatismo del parto y el puerperio son de consecuencias funestas. En tal situación reanudamos el estudio a partir de la 4a o 6a semana del puerperio, a base de citologías, biopsias, conización.

7. Aconsejamos una citología antes del parto; si es negativa puede permitirse el parto por las vías naturales. Se practicará cesárea cuando la citología post-conización o ante-partum resultare positiva o sospechosa.

8. Proponemos una conducta a seguir en la paciente embarazada de acuerdo al examen clínico y los hallazgos citológicos.

9. Presentamos nuestra casuística y su comentario. Existen conductas erradas debido a que aún no teníamos un criterio definido sobre este problema. De los 6 casos, 4 fueron descubiertos por medio de la citología vaginal rutinaria. Los casos conizados ninguno presentó complicación. 
10. Se presenta la bibliografía sobre tal tema.

\section{BIBLIOGRAFIA}

1 NESBIT R. E. L. Jr., HELLMAN L. M.: The histopatology and citology of the cervix in pregnancy. Surg. Gynec. \& Obst. 94. 1952.

2 ROVINSKY J. J.; GUTTMACHER A. F. Complicaciones Médicas, Quirúrgicas y Ginecológicas en el embarazo. Capítulo XXV. Citología Vaginal; por Nieburgs H. E. y Clyman M. Edit. Interamericana, México, 1967. Págs. 271-297.

3 FRITZGERALD, P. J., y HELLMAN. L. M. : Cervical changes in pregnancy and carcinoma in situ. New York. J. Med. 60: 3095, 1960.

4 FLUHMANN C. F.: El cuello uterino y sus enfermedades. Edición 1ra. 1963. Pág. 521.

5 BENSON, RALPH C.: Manual de Obstetricia y Ginecología. Edición 1ra. 1966. Pág. 517.

6 ROSENTHAL, A., y HELLMAN M.: The epithelial changes in the fetal cervix, includin the role of the "reserve cell". Am. J. Cbst. \& Gynec. 64: 260, 1952.

7 WESPI, H.: Early carcinoma of the uterine cervix. Grune \& Stratton, Inc. New York 1949.

8 EPPERSON, J. W., HELLMAN L. M., CALVIN, GA y BUSBY, T.: Am. J. Obst. \& Gynec. 61, 50, 1951.

9 NESBIT y cols.: The histopatology and citology of the cervix during pregnancy. Durg. Gynec. \& Obst. 94: 10, 1951.

10 NOVAK, JONES, JONES: Tratado de Ginecología. Edición 7a. 1966. Pág. 697.

11 DANFORTH, D. N.: Am. J. Obst. \& Gynec. 60: $985,1950$.

12 CAMPOS, J. y SOIHEIT, S.: Surg. Gynec. \& Obst. 102, 427, 1956.

13 HeLLMAN, L. M.: Progr. Gynec. 3, 433, 1957.

14 NESBIT R. E. L. Jr.: Obst. \& Gynec. 6, 239,1955

15 GREENE, R. R. y cols. y PECKMAN, B. M. : Am. J. Obst. \& Gynec. 75, 551, 1958.

16 HAMPERL, H., KAUFMANN C. y OBER, KG: Archiv. Gynak, 181, 184. 1954.

17 MARCUS. M. B., BRANDT, M. L., y $\mathrm{Cl}$ BLEY, L. J.: Obst. \& Gynec. 10, 669, 1957.
18 MARSH, M. y FRITZGERALD, P. J.: Cáncer. 9, 1195, 1956.

19 MOORE, D. B. y GUSBERG, S. B.: Obst. \& Gynec. 18, 530, 1959.

20 PECKHAM, B., GREENE, R. CHUNG. J. R. BAYLY, M. A. y BENARON. H. B. W.: Am. J. Obst. \& Gynec. 67, 21, 1954.

21 RICHARD R.: Am. J. Cbst. \& Gynec. 87, 474,1963

22 TE-LINDE R.: Ginecología operatoria. Ed. Bernades, S. A. 3ra. Edición. 1966. Pág. 480.

23 RAD M., MARCZINKE I., BOYES D. A., FIDLER H. K.: El uso de la citología exfoliativa vaginal durante el embarazo. Am. J. Obst. \& Gynec. Memorias de la XXIII de la Soc. de Obst. y Ginec. del Canadá. Vol. 94, No 4 Febrero de 1966.

24 SLATE, T. A., MARTIN P. L. Y MERRIT J. W.: Am. J. Obst. \& Gynec. 74, 344, 1957.

25 FERGUSON y CAVANAGH: Am. J. Obst. \& Gynec. 74, 344, 1957.

26 AYRE, J. E. Obst. \& Gynec. 76, 780, 1958.

27 SPUJT, H. J., RUCH, W. A., MARTIN, P. A., y HOBBS, J. E.: Obst. \& Gynec. 15, 19,1960

28 LULL y KIMBROUGH. Obstetricia Clínica. Editorial Interamericana S. A. 1960. Pág. 339.

29 SCHMITZ, H. E., ISAACS J. H. y FETHERSTON, W. C.: The value of routine cytologic. smears in pregnancy. Amer. J. Obst. \& Gynec. 79: 910, 1960.

30 LEON JUAN. Tratado de Obstetricia. Ediciones M. Segura. 2a. Edición. Vol. 2. Pág. 188

31 AREVALO B. EDUARDO y LOZANO A. : Embarazo y Malignidad. Rev. Obst. \& Gin. XVII. 41. 1966.

32 WILLIAMS E.: Obstetricia. Editorial Uteha. 3ra. Edición. 1960.

33 MONTGOMERY, T. L. Obst. \& Gynec. 3 : 268-276. Sept. 1953.

34 DEAN R. E., ISBELL N. P., WOODARD D. E. : Cervical ca in pregnancy. Obst. \& Gynec. 20: 635, 1952.

35 JORDAN G., DARNALT R. E.: Cáncer del cuello uterino y embarazo. Rev. Col. Obst. y Gin. Memorias de la 2a. Convención Col. de Obst. y Gin. Nov.-Dic. de 1955. 
36 DARNALT R. E.: Conización del cuello uterino. Rev. Col. de Obst. y Gin. XVI. № 6. 1966.

37 OSBAND R., NICHOLSON JONES W.: Ca in situ in pregnancy. Am. J. Obst. Gynec. 83: 605,1952 .

33 PARKER, R. T. y cols.: Am. J. Obst. \& Gynec. 80: 693, 1960.

39 FESTE J., KAUFMANN R., SKCGLAND H., TOPEK, N.: Obst. \& Gynec. 95: 763, 1966.

40 GALLEgOS y cols.: Ca in situ. Rev. Obst. y Gin. de Venezuela. 27: 271, 1967. pág. 271.

41 GONZALEZ LOYA y cols.: Ca intraepitelial. Revisión de 31 casos. Rev. de Obst. y Gin. de México. XXII. № 33, 1961.

42 FERGUSON J.: Cervical conization during pregnancy. Surg. Obst. \& Gynec. 111, 5: $603,1960$.

43 MOORE, G., WELLS: Management of superficial cancer in pregnancy. Obst. \& Gynec. 27: $307,1966$.
44 MUSSEY, E., DECKER, D. G.: Intraepitelial carcinoma of the cervix in association with pregnancy. Am. J. Obst. \& Gynec. 97: 1, 30,1967

45 TAYLOR, H. S.: The management of in situ carcinoma of the cervix. Rev. Inst. Nac. Cancerol. 16: 407, 1964.

46 OFFENN J. H., y FERGUSON J. H.: Cold conization of the cervix; a practical technic for maximum hemostasis. Obst. \& Gynec. $15: 396,1960$.

47 ROVINSKY J. J., GUTTMACHER A. F. Complicaciones Médicas, Quirúrgicas y $\mathrm{Gi}$ necológicas en el embarazo. Capítulo 26: Carcinoma del cuello uterino, por Lapid L. S., Davids A. M., Gusber S. B. Edit. Interamericana; México, 1967. Págs. 298306.

48 KAPLAN A. L., KAUFMAN R. H.: Diagnóstico y tratamiento de la displasia y el carcinoma in situ del cuello uterino durante la gestación. Clínicas Obstétricas y Ginecológicas. Diciembre de 1967. 